\title{
VLT/UVES shows no cosmological variability of $\alpha$
}

\author{
Paolo Molaro ${ }^{1}$, Miriam Centurion ${ }^{1}$, Sandro D'Odorico ${ }^{2}$, and \\ Sergei Levshakov ${ }^{3}$ \\ ${ }^{1}$ Osservatorio Astronomico di Trieste-INAF, Via G.B. Tiepolo 11 I-34131, Trieste, Italy \\ ${ }^{2}$ ESO, Karl-Schwarzschild-Strasse 2 D-85748 Garching bei Munchen, Germany \\ ${ }^{3}$ Ioffe Physico-Technical Institute, St. Petersburg, Russia
}

\begin{abstract}
The cosmological variability of $\alpha$ is probed from individual observations of pairs of Fe II lines. This procedure allows a better control of the systematics and avoids the influence of the spectral shifts due to ionisation inhomogeneities in the absorbers and/or non-zero offsets between different exposures. Applied to the Fe II lines of the metal absorption systems at $z_{\text {abs }}=$ 1.839 in Q1101-264 and at $z_{\text {abs }}=1.15$ in HE0515-4414 observed by means of UVES at the ESO-VLT, it provides $\Delta \alpha / \alpha=\left(0.4 \pm 1.5_{\text {stat }}\right) \times 10^{-6}$. The result is shifted with respect to the Keck/HIRES mean $\Delta \alpha / \alpha=\left(-5.7 \pm 1.1_{\text {stat }}\right) \times 10^{-6}$ (Murphy et al. 2004) at a high confidence level (95\%). Full details of this work are given in Levshakov et al. (2005).
\end{abstract}

\section{Introduction}

The Sommerfeld fine-structure constant $\alpha \equiv e^{2} / \hbar c$ describes electromagnetic and optical properties of atoms and is the most suitable constant for time variation tests in the lab or for astronomical observations (for a review see Uzan 2003). The value is $\alpha=1 / 137.03599976(50)$ (Mohr \& Tailor 2000) and laboratory experiments constrain its variability as $d \ln [\alpha(t)] / d t=\left(-0.9 \pm 2.9_{\text {stat }}\right) \times 10^{-15} \mathrm{yr}^{-1}$ (Fischer et al. 2004). This limit corresponds to $|\Delta \alpha / \alpha| \equiv\left|\left(\alpha_{z}-\alpha\right) / \alpha\right|<3.8 \times 10^{-5}$ for $t \sim 10^{10}$ yr and $\alpha_{z}$ varying linearly with time, which may be not the case. Analysis of radioactive decay rates of nuclei in meteorites set $\Delta \alpha / \alpha=\left(+8 \pm 8_{\text {stat }}\right) \times 10^{-7}$ (Olive et al. 2004) while a recent analysis of the isotopic abundances in the Oklo samples $\left(\Delta t \sim 2 \times 10^{9} \mathrm{yr}\right)$ suggests $\Delta \alpha / \alpha \geqslant 4.5 \times 10^{-8}$ (Lamoreaux \& Torgerson 2004). Shifts in the alkali doublets provide $\Delta \alpha / \alpha=\left(-0.5 \pm 1.5_{\text {stat }}\right) \times 10^{-5}$ (Murphy et al. 2001) but the ManyMultiplet method applied to 143 absorption systems in the Keck/HIRES spectra of quasars indicates a decrease of $\alpha: \Delta \alpha / \alpha=\left(-5.7 \pm 1.1_{\text {stat }}\right) \times 10^{-6}$ in the redshift range $0.2<z<4.2$ (Murphy et al. 2004, MFWDPW). However, VLT/UVES observations of 23 absorption systems $(0.4 \leqslant z \leqslant 2.3)$ toward 18 QSOs show no variability: $\Delta \alpha / \alpha=\left(-0.6 \pm 0.6_{\text {stat }}\right) \times 10^{-6}$ (Chand et al. 2004). A first result of the new approach presented here applied to the system at $z_{\mathrm{abs}}=1.15$ towards HE0515-4414 $(B=15.0)$ was $\Delta \alpha / \alpha=\left(-0.4 \pm 1.9_{\text {stat }} \pm 2.7_{\text {sys }}\right) \times 10^{-6}$ (Quast, Reimers \& Levshakov 2004), also showing no variability of $\alpha$.

\section{The concept}

We use a modified Many-Multiplet procedure to calculate $\Delta \alpha / \alpha$, directly from the differences between the wavelengths of a pair of Fe II transitions observed in the individual 
exposures. We call it SIDAM Single Ion Differential $\alpha$ Measurement. Full details of the method and of the analysis presented here are given in Levshakov et al. (2005).

- Why FeII ?

Dealing with only one heavy element we reduce: $(i)$ the influence of unknown isotopic ratios. For Fe II this is less pronounced than that for MgII because iron is heavier and its isotope structure is more compact. The relative abundance of the leading isotope ${ }^{56} \mathrm{Fe}$ is higher (terrestrial isotope ratios are ${ }^{54} \mathrm{Fe}:{ }^{56} \mathrm{Fe}:{ }^{57} \mathrm{Fe}:{ }^{58} \mathrm{Fe}=5.8: 91.8: 2.1: 0.3$, and $\left.{ }^{24} \mathrm{Mg}:{ }^{25} \mathrm{Mg}:{ }^{26} \mathrm{Mg}=79: 10: 11\right)$. (ii) The effects of possible inhomogeneous ionisation structure within the absorber. (iii) For Fe II the relativistic correction to the changes in $\alpha$ ( $q$ coefficients) are rather large.

- Why single exposures?

Being applied to the lines from the same exposure, this method does not depend on the unknown offsets of the wavelength scale. There are three main sources of potential systematic errors in the absolute velocity scale: $(i)$ temperature, $(i i)$ air pressure instability, and (iii) mechanical instabilities of unknown origin. For instance, a change of 1 millibar, or a change of $0.3^{\circ} \mathrm{C}$, corresponds to an error in radial velocities of $\sim 50 \mathrm{~m} \mathrm{~s}^{-1}$, or $\Delta \alpha / \alpha \sim 1.7 \times 10^{-6}$.

\section{Observations and data reduction}

Five high resolution UVES spectra of Q1101-264 $(V=16.02)$ with a $z_{\text {abs }}=1.839$ system were obtained with a dichroic filter during the Science Verification programme in February 2000. The spectral resolution as measured from the ThAr emission lines are of $\mathrm{FWHM} \simeq 6.0 \mathrm{~km} \mathrm{~s}^{-1}$ in the blue $(\lambda \sim 4570 \AA)$, and of $\simeq 5.4 \mathrm{~km} \mathrm{~s}^{-1}$ in the red $(\lambda \sim 7380 \AA)$. We modified the UVES pipeline to calibrate the echelle spectra without re-binning in wavelength to preserve the original pixel size: $50 \mathrm{~m} \AA\left(3.3 \mathrm{~km} \mathrm{~s}^{-1} / \mathrm{pix}\right)$ for the blue and $55 \mathrm{~m} \AA\left(2.2 \mathrm{~km} \mathrm{~s}^{-1} /\right.$ pix $)$ for the red. The typical $\mathrm{rms}$ of the wavelength calibration were $\approx 1 \mathrm{~m} \AA\left(\approx 60 \mathrm{~m} \mathrm{~s}^{-1}\right)$. We worked with individual exposures, since co-adding them cancels out original non-zero offsets and perturbs line centroids. All spectra are corrected to heliocentric and vacuum scale but they are not combined and not re-sampled.

\section{Discussion}

Measurements for Q1101-264 were combined with the Fe II sample from the $z_{\text {abs }}=1.15$ system toward HE0515-4414 (QRL) providing a total of $35 \Delta \alpha / \alpha$ values. The normalised distribution of the resulting $35 \Delta \alpha / \alpha$ values is plotted with a histogram in Fig. 1 . In the figure the results of MFWDPW and CSPA are also shown by the dashed and dotted curves, respectively, assuming that the measured $\Delta \alpha / \alpha$ are normally distributed with the sample means and standard deviations taken from the original papers. The vertical lines in this figure mark the centres of the corresponding distributions. Our result is shown by the histogram and has the sample mean:

$$
\langle\Delta \alpha / \alpha\rangle=\left(0.4 \pm 1.5_{\text {stat }}\right) \times 10^{-6} .
$$

The scatter of $\Delta \alpha / \alpha$ in the Keck sample is about 2 times the $\sigma_{\text {rms }}$ value of our combined Fe II sample, but the sample means of CSPA and our Fe II ensemble are in good agreement. The results presented in Fig. 1 show that $\langle\Delta \alpha / \alpha\rangle_{\text {Keck/HIRES }} \neq\langle\Delta \alpha / \alpha\rangle_{\text {VLT } / \text { UVES }}$. Our result, and also the one of CSPA, differ from that of MFWDPW at a $95 \%$ significance level according to the $t$-test. 


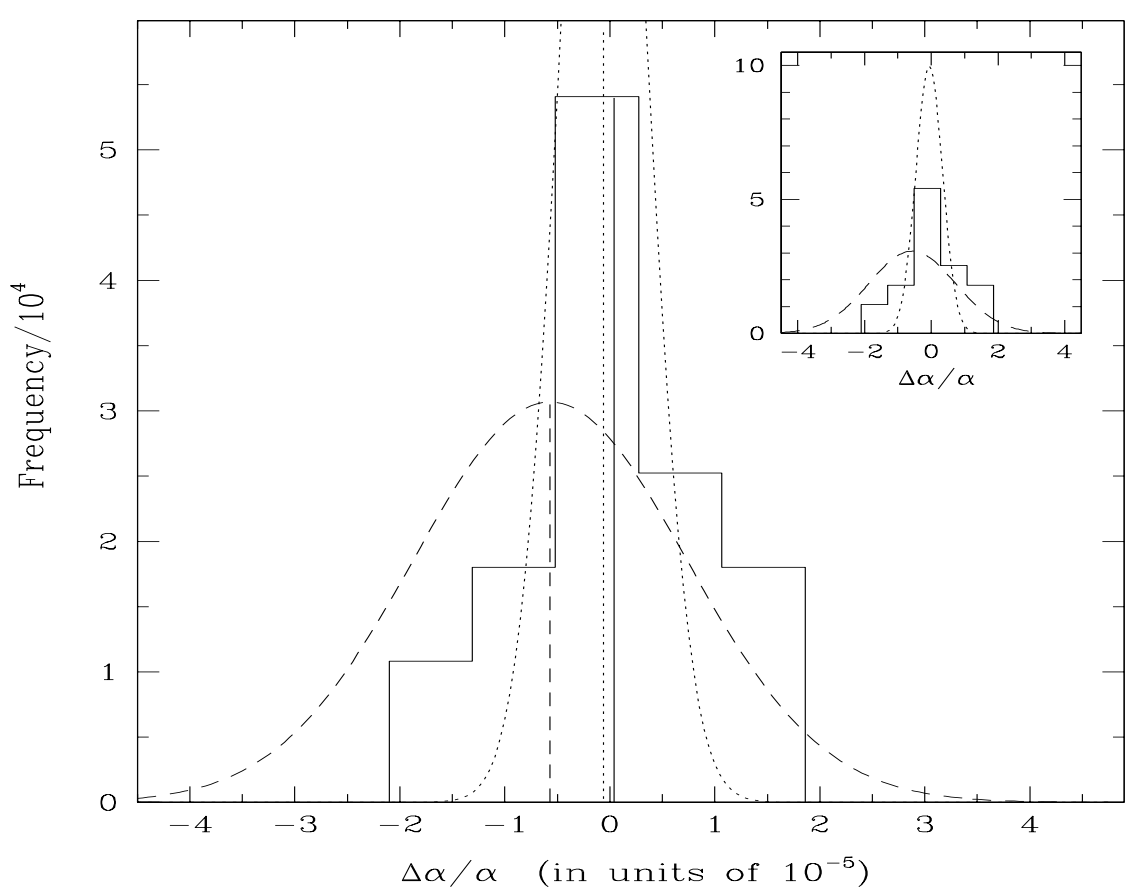

Figure 1. The histogram shows the distribution of $\Delta \alpha / \alpha$ from the Fe II systems in Q1101-264 and HE0515-4414. The dashed curves show the distributions from MFWDPW and CSPA. Note the discrepancy between Keck/HIRES and VLT/UVES sample mean values.

We also note that the standard deviation in the CSPA sample is exceptionally small, somewhat in disagreement with their wavelength calibration accuracy. This was checked through the relative velocity shifts, $\Delta v$, between the Fe II $\lambda 2344$ and $\lambda 2600$ lines. The mean $\langle\Delta v\rangle$ shows a dispersion of $\sigma_{\Delta v} \simeq 0.4 \mathrm{~km} \mathrm{~s}^{-1}$, equivalent to $\sigma_{\Delta \alpha / \alpha} \sim 2 \times 10^{-5}$, which is not consistent with an error of the mean $\sigma_{\langle\Delta \alpha / \alpha\rangle} \sim 0.6 \times 10^{-6}$ as CSPA claimed.

Although the result presented here is consistent with no evolution of $\alpha$, we emphasise that accurate individual measurements of $\langle\Delta \alpha / \alpha\rangle$ are required if $\alpha$ has an oscillating behaviour as suggested by Fuji (2005).

\section{References}

Bahcall, J. N., Steinhardt, C. L., Schlegel, D., 2004, ApJ, 600, 520 [BSS]

Chand, H., Srianand, R., Petitjean, P., Aracil, B., 2004, A\&A, 417, 853 [CSPA]

Fuji, A., 2005, astro-ph/0502191

Lamoreaux, S. K., Torgerson, J. R., 2004, Phys. Rev. D, 69, 121701

Levshakov, S. A., 2004, in Astrophysics, Clocks, and Fundamental Constants. eds. S. G. Karshenboim \& E. Peik (Springer-Verlag: Berlin, Heidelberg), p. 151

Levshakov, S. A. Centurion, M., D'Odorico, S., Molaro, P., 2005, A\&A, in press, astroph-0408188 Murphy, M. T., Flambaum, V. V., Webb, J. K., Dzuba, V. V., Prochaska, J. X., Wolfe, A. M., 2004, in Astrophysics, Clocks, and Fundamental Constants, eds. S. G. Karshenboim \& E. Peik (Springer-Verlag: Berlin, Heidelberg), p. 131 [MFWDPW]

Murphy, M. T., Webb, J. K., Flambaum, V. V., 2003, MNRAS, 345, 609

Murphy, M. T., Webb, J. K., Flambaum, V. V., Prochaska, J. X., Wolfe, A. M., 2001, MNRAS, 327,1237

Olive, K. A., Pospelov, M., Qian, Y.-Z., et al., 2004, Phys. Rev. D, 69, 027701

Quast, R., Reimers, D., Levshakov, S. A., 2004, A\&A, 415, L7 [QRL] 